\title{
Erratum to: Modeling the photosynthetically active radiation in South West Amazonia under all sky conditions
}

\author{
Leonardo J. G. Aguiar • Graciela R. Fischer •
}

Richard J. Ladle • Ana C. M. Malhado •

Flávio B. Justino • Renata G. Aguiar •

José Maria N. da Costa

Published online: 14 March 2012

(C) Springer-Verlag 2012

\section{Erratum to: Theor Appl Climatol}

DOI 10.1007/s00704-011-0556-Z

In the original paper the coefficients of the linear regressions in Tables 1 and 2 were not placed in the correct order. The correct ones are as follows:

The online version of the original article can be found at http://dx.doi. org/10.1007/s00704-011-0556-z.

L. J. G. Aguiar $(\bowtie) \cdot$ G. R. Fischer $\cdot$ F. B. Justino

J. M. N. da Costa

Department of Agricultural and Environmental Engineering,

Federal University of Vicosa,

Av. P. H. Rolfs,

Vicosa, Minas Gerais, Brazil 36570-000

e-mail: veraneiro@yahoo.com.br

R. J. Ladle · A. C. M. Malhado

Institute of Biological and Health Sciences,

Federal University of Alagoas,

Maceió, Alagoas, Brazil

R. J. Ladle

School of Geography and the Environment, University of Oxford,

Oxford, UK

R. G. Aguiar

Department of Environmental Engineering,

Federal University of Rondonia,

Ji-Parana, Rondonia, Brazil 
1. Table 1 should read:

Table 1

\begin{tabular}{|c|c|c|c|c|c|c|c|}
\hline Period & Model & $a$ & $b$ & $c$ & $d$ & $R^{2}$ & $N$ \\
\hline \multicolumn{8}{|l|}{ Pasture } \\
\hline \multirow[t]{3}{*}{ Wet season } & Mod. 1 & 0.474 & 0.283 & & & 0.999 & 2171 \\
\hline & Mod. 2 & 0.478 & -5.547 & 1.146 & & 0.999 & 2167 \\
\hline & Mod. 3 & 0.478 & -6.088 & -0.385 & 11.196 & 0.999 & 2167 \\
\hline \multirow[t]{3}{*}{ Wet-dry season } & Mod. 1 & 0.467 & -0.818 & & & 0.998 & 2094 \\
\hline & Mod. 2 & 0.471 & -6.911 & 1.336 & & 0.998 & 2079 \\
\hline & Mod. 3 & 0.470 & -6.511 & 0.246 & -4.534 & 0.998 & 2079 \\
\hline \multirow[t]{3}{*}{ Dry season } & Mod. 1 & 0.449 & -4.192 & & & 0.993 & 2208 \\
\hline & Mod. 2 & 0.445 & 7.669 & -6.633 & & 0.993 & 2197 \\
\hline & Mod. 3 & 0.444 & 10.533 & 0.339 & -14.164 & 0.994 & 2197 \\
\hline \multirow[t]{3}{*}{ Dry-wet season } & Mod. 1 & 0.464 & -1.160 & & & 0.998 & 1954 \\
\hline & Mod. 2 & 0.480 & -27.641 & 3.904 & & 0.998 & 1954 \\
\hline & Mod. 3 & 0.480 & -27.739 & -0.127 & 7.102 & 0.998 & 1954 \\
\hline \multirow[t]{3}{*}{ Annual } & Mod. 1 & 0.462 & -1.162 & & & 0.995 & 8427 \\
\hline & Mod. 2 & 0.469 & -11.761 & 1.612 & & 0.995 & 8397 \\
\hline & Mod. 3 & 0.464 & -3.210 & 1.105 & -26.505 & 0.996 & 8397 \\
\hline \multicolumn{8}{|l|}{ Forest } \\
\hline \multirow[t]{3}{*}{ Wet season } & Mod. 1 & 0.423 & -0.407 & & & 0.993 & 2076 \\
\hline & Mod. 2 & 0.437 & -22.882 & 3.074 & & 0.994 & 2067 \\
\hline & Mod. 3 & 0.441 & -27.446 & 0.686 & -12.054 & 0.994 & 2049 \\
\hline \multirow[t]{3}{*}{ Wet-dry season } & Mod. 1 & 0.420 & 0.766 & & & 0.996 & 1283 \\
\hline & Mod. 2 & 0.424 & -8.042 & 3.121 & & 0.996 & 1283 \\
\hline & Mod. 3 & 0.425 & -8.972 & 0.436 & -6.610 & 0.996 & 1283 \\
\hline \multirow[t]{3}{*}{ Dry season } & Mod. 1 & 0.432 & -3.444 & & & 0.994 & 898 \\
\hline & Mod. 2 & 0.436 & -9.302 & -0.807 & & 0.994 & 898 \\
\hline & Mod. 3 & 0.435 & -4.325 & 0.606 & -15.891 & 0.994 & 898 \\
\hline \multirow[t]{3}{*}{ Dry-wet season } & Mod. 1 & 0.427 & 0.594 & & & 0.993 & 1087 \\
\hline & Mod. 2 & 0.447 & -32.168 & 5.544 & & 0.994 & 1087 \\
\hline & Mod. 3 & 0.447 & -34.066 & 0.274 & 0.518 & 0.994 & 1087 \\
\hline \multirow[t]{3}{*}{ Annual } & Mod. 1 & 0.425 & -0.482 & & & 0.993 & 5344 \\
\hline & Mod. 2 & 0.434 & -16.509 & 2.939 & & 0.994 & 5335 \\
\hline & Mod. 3 & 0.435 & -18.125 & 0.467 & -7.072 & 0.994 & 5317 \\
\hline
\end{tabular}


2. Table 2 should read:

Table 2

\begin{tabular}{|c|c|c|c|c|c|c|c|}
\hline Period & Model & $a$ & $b$ & $c$ & $d$ & $R^{2}$ & $N$ \\
\hline \multicolumn{8}{|l|}{ Pasture } \\
\hline \multirow[t]{3}{*}{ Wet season } & Mod. 1 & 0.464 & 3.847 & & & 0.998 & 181 \\
\hline & Mod. 2 & 0.466 & -1.516 & 3.847 & & 0.998 & 181 \\
\hline & Mod. 3 & 0.466 & -1.362 & -0.023 & 4.447 & 0.998 & 181 \\
\hline \multirow[t]{3}{*}{ Wet-dry season } & Mod. 1 & 0.459 & 2.363 & & & 0.996 & 174 \\
\hline & Mod. 2 & 0.513 & -39.827 & 2.737 & & 0.997 & 174 \\
\hline & Mod. 3 & 0.487 & -22.223 & 0.499 & -8.390 & 0.998 & 174 \\
\hline \multirow[t]{3}{*}{ Dry season } & Mod. 1 & 0.449 & -4.044 & & & 0.946 & 184 \\
\hline & Mod. 2 & 0.335 & 79.082 & -0.946 & & 0.959 & 184 \\
\hline & Mod. 3 & 0.347 & 86.718 & 0.927 & -27.462 & 0.969 & 184 \\
\hline \multirow[t]{3}{*}{ Dry-wet season } & Mod. 1 & 0.447 & 5.389 & & & 0.995 & 162 \\
\hline & Mod. 2 & 0.709 & -234.375 & 4.109 & & 0.996 & 162 \\
\hline & Mod. 3 & 0.766 & -286.430 & -0.629 & 20.423 & 0.997 & 162 \\
\hline \multirow[t]{3}{*}{ Annual } & Mod. 1 & 0.443 & 6.254 & & & 0.976 & 701 \\
\hline & Mod. 2 & 0.479 & -27.375 & 5.257 & & 0.977 & 701 \\
\hline & Mod. 3 & 0.435 & 17.944 & 1.495 & -34.302 & 0.988 & 701 \\
\hline \multicolumn{8}{|l|}{ Forest } \\
\hline \multirow[t]{3}{*}{ Wet season } & Mod. 1 & 0.416 & 1.881 & & & 0.984 & 175 \\
\hline & Mod. 2 & 0.457 & -41.467 & 2.738 & & 0.985 & 175 \\
\hline & Mod. 3 & 0.453 & -40.867 & 0.903 & -16.421 & 0.991 & 174 \\
\hline \multirow[t]{3}{*}{ Wet-dry season } & Mod. 1 & 0.422 & -0.154 & & & 0.995 & 109 \\
\hline & Mod. 2 & 0.410 & 9.599 & -0.339 & & 0.995 & 109 \\
\hline & Mod. 3 & 0.418 & 3.108 & 0.396 & -8.834 & 0.996 & 109 \\
\hline \multirow[t]{3}{*}{ Dry season } & Mod. 1 & 0.433 & -4.017 & & & 0.947 & 77 \\
\hline & Mod. 2 & 0.431 & 1.893 & -4.142 & & 0.947 & 77 \\
\hline & Mod. 3 & 0.442 & 9.893 & 1.069 & -35.878 & 0.957 & 77 \\
\hline \multirow[t]{3}{*}{ Dry-wet season } & Mod. 1 & 0.422 & 2.672 & & & 0.989 & 92 \\
\hline & Mod. 2 & 0.384 & 39.020 & 2.128 & & 0.989 & 92 \\
\hline & Mod. 3 & 0.396 & 17.392 & 0.773 & -10.179 & 0.992 & 92 \\
\hline \multirow[t]{3}{*}{ Annual } & Mod. 1 & 0.421 & 0.795 & & & 0.985 & 453 \\
\hline & Mod. 2 & 0.426 & -4.352 & 0.925 & & 0.985 & 453 \\
\hline & Mod. 3 & 0.426 & -6.702 & 0.703 & -13.566 & 0.988 & 452 \\
\hline
\end{tabular}

